'post'. In brief, the major finding was that whereas the inhibitory input conductance was similar for the two directions of motion, the excitatory input conductance differed significantly for preferred and null direction motion: the excitatory input to the turtle ganglion cell is already directionally selective! The author even goes one step further: by constructing a biophysically realistic model of the ganglion cell, he implemented a postsynaptic mechanism ${ }^{3}$, and then simulated the experiments he was going to do on the real ganglion cells. He thus obtained an expectation of what he should see if the postsynaptic 'shunting inhibition' model were true. The prediction that came out of this modeling was that the overall conductance change observed during null direction motion should, first, outlast the one observed during preferred direction motion, and, second, be almost twice as large as the resting conductance of the neuron. Otherwise, the inhibitory input would not effectively shunt the excitatory input. Both of these predictions failed. The postsynaptic shunting inhibition model thus was killed twice: not only was the inhibitory conductance insufficient to produce any sort of direction selectivity in the ganglion cell, but also the excitatory input was already directionally selective. So, the answer we are given by Borg-Graham is clear-cut: it's pre.

This would be the end of a longstanding debate, if not for a paper pub- lished a few months ago stating just the opposite conclusion ${ }^{4}$. Applying an experimental protocol similar to BorgGraham (whole-cell patch recording under voltage-clamp conditions), Taylor et al. investigated the same phenomenon in retinal ganglion cells of the rabbit. By increasing the internal chloride concentration, and thus shifting the equilibrium (reversal) potential for chloride toward $0 \mathrm{mV}$ (making inhibitory and excitatory inputs both produce inward currents), the authors were able to completely eliminate direction-selective responses. Because this should not have any effect on an input that is already directionally selective, this finding was hard to reconcile with a presynaptic mechanism. Based on this and other experiments, the conclusion drawn by the authors was that it's post.

What are we left with? There are several possibilities to consider. Because both studies were carried out on different species, no factual discrepancy exists so far. It might well be that rabbits and turtles indeed have found different solutions to the same problem in evolution, so that the final answer will be turtles pre, rabbits post. This should be relatively easy to test by the different research teams. The other possibility is that the process creating direction selectivity is a local one, and some aspects of its operation simply escape the reach of the electrode in whole-cell protocols.
Such phenomena exist ${ }^{5,6}$, and looking at the intricate mesh of synapses formed in the inner plexiform layer by glutamatergic terminals of bipolar cells, amacrine cells synapses co-releasing acetylcholine and $\mathrm{GABA}^{7}$, and ganglion cell dendrites, we realize that the substrate for local circuits exists there in plenty. Finally, the search for 'the' mechanism underlying direction selectivity, even in the retina of one species, could be hampered by the existence of a multi-step process $^{8}$, with the spike threshold of the ganglion cell being the final step in enhancing direction selectivity. In conclusion, while the present study marks an important step forward, there still remains a big nut to be cracked-so stay tuned!

1. Borg-Graham, L. Nat. Neurosci. 4, 176-183 (2001).

2. Barlow, H. B. \& Levick, W. R. J. Physiol (Lond). 178, 477-504 (1965).

3. Koch, C., Poggio, T. \& Torre, V. Proc. Natl. Acad. Sci. USA 80, 2799-2802 (1983).

4. Taylor, W. R., He, S., Levick, W. R. \& Vaney, D. I. Science 289, 2347-2350 (2000).

5. Coleman, M. J., Meyrand, P. \& Nusbaum, M. P. Nature 378, 502-505 (1995).

6. Debanne, D., Guerineau, N. C., Gähwiler, B. H. \& Thompson, S. M. Nature 389, 286-289 (1997).

7. O'Malley, D. \& Masland, R. H. Proc. Natl. Acad. Sci. USA 86, 3414-3418 (1989).

8. Borst, A. \& Egelhaaf, M. Proc. Natl. Acad. Sci. USA 87, 9363-9367 (1990).

9. Torre, V. \& Poggio, T. Proc. R. Soc. Lond. B 202, 409-416 (1978).

\title{
Going with the (virtual) flow
}

In theory, navigation toward a goal could be guided by several visual cues. The direction of a goal could be calculated with respect to the body (the 'egocentric direction hypothesis'). Alternatively, people might move in the direction that minimizes the error between cues from the expanding radial pattern on the retina ('optic flow' is produced by self-movement) and the goal. In practice, these two strategies for visual control are difficult to distinguish because they predict the same behavior. However, Warren and colleagues from Brown University (page 213) created a virtual reality environment that allows their subjects to walk through a world where the laws of optics are under experimental control. The authors can create conditions that never occur in the natural world, such as displacing the optic flow field from the actual direction of walking. In such a virtual world, the egocentric direction and optic flow hypotheses make different predictions about the shape of a subject's path toward a goal. The authors found that when little optic flow information was available, subjects' behavior was consistent with the egocentric direction hypothesis. However, when the environment was made more complex, for example by adding textured floors and ceilings, optic flow information increasingly dominated behavior. These results demonstrate that the visual system can control locomotion robustly under a variety of environmental conditions, and that optic flow cues are used to control human walking when they are available.

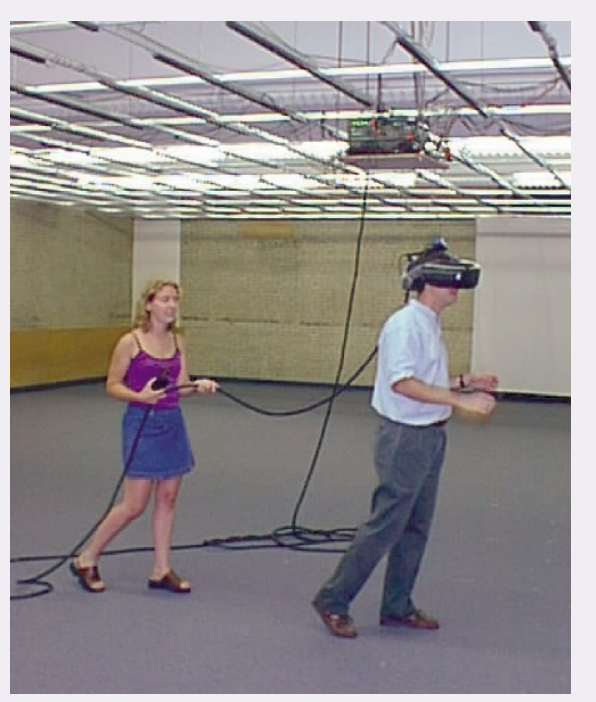

John E. Spiro 